\title{
The Vorticity-Velocity Gradient Cofactor Tensor and the Material Invariant of the Semigeostrophic Theory
}

\author{
Álvaro Viúdez \\ Institut de Ciències del Mar, CSIC, Barcelona, Spain
}

(Manuscript received 1 September 2004, in final form 5 December 2004)

\begin{abstract}
A new derivation and interpretation of the semigeostrophic (SG) material invariant in the theory of geophysical flows is introduced. First, a generalized three-dimensional equation of the SG dynamics is established and the generalized equations for the rate of change of vorticity and for the rate of change of the velocity gradient cofactor tensor are obtained. Next, a conservation equation for the vorticity-velocity gradient cofactor tensor (denoted $\tilde{\Xi}$ ) is derived. The specific potential $\tilde{\Xi}$, that is, $\tilde{\Xi}$ in the reference configuration per unit of mass, is defined and an expression for its rate of change is obtained. The SG invariant is interpreted as the vertical component of the specific potential $\Xi$. Under the SG assumptions (advection of the geostrophic velocity, hydrostatic, and $f$-plane approximations) this vertical component is materially conserved in the SG flow. The generalized SG invariant (i.e., the specific potential $\Xi$ ) differs conceptually from the Beltrami-Rossby-Ertel specific potential vorticity. Its conservation in the SG flow seems to be highly dependent on the SG assumptions, especially on the f-plane approximation and on the horizontal nature of the geostrophic velocity.
\end{abstract}

\section{Introduction}

In the semigeostrophic (SG) theory of geophysical flows (Eliassen 1949; Fjortoft 1962; Hoskins 1975; Hoskins and Draghici 1977) the horizontal acceleration relative to the rotating frame is replaced by the rate of change of the horizontal geostrophic velocity. Under the hydrostatic and $f$-plane approximations the SG flow has a scalar material invariant (Hoskins 1975) commonly referred to as the SG potential vorticity (PV). The name SG PV was chosen due to its similarity to Rossby-Ertel PV (Rossby 1940; Ertel 1942; Schröder 1991), also known as Beltrami's material vorticity (Beltrami 1871; Casey and Naghdi 1991; Viúdez 2001). Recently Bridges et al. (2005) provided, in the language of differential forms (coordinate free), the relationship between the SG PV and the symplectic form of the Hamiltonian system for the SG shallow-water dynamics. Also recently, Ehrendorfer (2004) achieved a new vector derivation of the SG invariant that depends on the SG assumptions, that is, using the geostrophic velocity and adopting the hydrostatic and $f$-plane approximations. However, the precise mathematical meaning of the SG invariant seems to remain unclear, in part

Corresponding author address: Dr. Álvaro Viúdez, Institut de Ciències del Mar, CSIC, P. Maritim 37-49, 08003 Barcelona, Spain.

E-mail: aviudez@cmima.csic.es because a generalized (independent of the SG assumptions) and coordinate-independent derivation and interpretation is apparently missing. The aim of this work is to provide such a generalized derivation and interpretation of the SG invariant.

First, a generalized three-dimensional equation of the SG dynamics is introduced and the equations for the rate of change of vorticity and for the rate of change of the velocity gradient cofactor tensor are derived (section 2a). Secondly, an equation for the rate of change of the vorticity-velocity gradient cofactor tensor (denoted $\tilde{\Xi}$ ) is obtained. The specific potential $\tilde{\Xi}$, that is, $\tilde{\Xi}$ in the reference configuration per unit of mass, is defined and an expression for its rate of change is derived (section 2b). Section $2 c$ shows that the SG invariant is the vertical component of the specific potential $\tilde{\Xi}$, and that this vertical component is conserved in the SG flow under the SG assumptions. Finally, section 3 provides useful mathematical identities and alternative derivations of the main equations given in section 2, and addresses the role of the geostrophic coordinates in the derivation of the SG invariant.

\section{The generalized SG invariant}

\section{a. Basic equations}

First, we consider the following generalized threedimensional equation of the SG dynamics: 


$$
\frac{d \tilde{\mathbf{u}}}{d t}+\boldsymbol{\omega}^{p} \times \mathbf{u}=\boldsymbol{\varphi}
$$

The material rate of change of any quantity $\chi$ in the SG flow is expressed as $d \chi / d t=\dot{\chi}=\partial \chi / \partial t+\mathbf{u} \cdot \nabla \chi$, where $\mathbf{u}=(u, v, w)$ is the SG velocity and $\boldsymbol{\nabla}$ is the threedimensional gradient operator. Components of vectors always refer here to a Cartesian coordinate system. The physical meaning of the vector $\tilde{\mathbf{u}}=(\tilde{u}, \tilde{v}, \tilde{w})$, having physical dimensions of velocity, is, at this stage, left arbitrary. In the SG theory the horizontal vector $\tilde{\mathbf{u}}_{h}$ is the geostrophic velocity $\mathbf{u}_{h}^{g}$, defined as a rotation of the horizontal pressure (geopotential) gradient. This geostrophic definition will be adopted later in section $2 \mathrm{c}$. The planetary vorticity $\boldsymbol{\omega}^{p}=\left(\omega^{p}, \eta^{p}, \zeta^{p}\right)=2 \boldsymbol{\Omega}$, with $\boldsymbol{\Omega}$ the planetary angular velocity, is a constant vector. At this stage there is no need to assume a particular coordinate system nor that the scalar components of $\boldsymbol{\omega}^{p}$ are constant (as in the $f$-plane approximation). Vector $\boldsymbol{\varphi}=$ $(\varphi, \psi, \phi)$ is a generic force vector (per unit of mass), left also undefined.

It is assumed that the SG flow conserves mass,

$$
\dot{\rho}+\rho \boldsymbol{\nabla} \cdot \mathbf{u}=0,
$$

where $\rho$ is the mass density. Therefore the fluid may be compressible. The existence of a scalar quantity $\theta$, later associated with some material invariant (specific entropy or potential temperature) is also assumed. From the basic equation of the generalized SG dynamics (1), mass conservation (2), and the existence of $\theta$ we derive below the equations for the rate of change of the vorticity-velocity gradient cofactor tensor $\widetilde{\Xi}$ and the rate of change of the specific potential $\tilde{\Xi}$.

\section{b. The rate of change of the vorticity-velocity gradient cofactor tensor}

\section{1) RATE OF CHANGE OF THE VORTICITY $\tilde{\boldsymbol{\omega}}$}

From the curl of (1) we obtain first the rate of change of the vorticity of $\tilde{\mathbf{u}}$, denoted $\tilde{\boldsymbol{\omega}} \equiv \boldsymbol{\nabla} \times \tilde{\mathbf{u}}$ (appendix A):

$$
\begin{aligned}
\frac{d \tilde{\boldsymbol{\omega}}}{d t}= & -\left(\tilde{\boldsymbol{\omega}}+\boldsymbol{\omega}^{p}\right) \boldsymbol{\nabla} \cdot \mathbf{u}+\nabla \mathbf{u}^{\mathrm{T}} \cdot\left(\tilde{\boldsymbol{\omega}}+\boldsymbol{\omega}^{p}\right)+\nabla \tilde{\mathbf{u}} \dot{\times} \boldsymbol{\nabla u} \\
& +\boldsymbol{\nabla} \times \boldsymbol{\varphi} .
\end{aligned}
$$

The first, second, and last terms on the rhs above are the analogous divergence, twisting, and curl terms of the usual vorticity equation. The reason for writing the twisting term as $\nabla \mathbf{u}^{T} \cdot\left(\tilde{\boldsymbol{\omega}}+\boldsymbol{\omega}^{p}\right)$, where superscript $\mathrm{T}$ means the transpose, instead of $\left(\tilde{\boldsymbol{\omega}}+\boldsymbol{\omega}^{p}\right) \cdot \nabla \mathbf{u}$ will become apparent later.

The new term is $\nabla \tilde{\mathbf{u}} \dot{\times} \boldsymbol{\nabla} \mathbf{u}$. Given two tensors of second rank $\mathbf{A}=A_{i j} \mathbf{e}_{i} \mathbf{e}_{j}$ and $\mathbf{B}=B_{m n} \mathbf{e}_{m} \mathbf{e}_{n}$ (Gibbsian dyadic notation for tensors is used and summation convention for repeated subindices is assumed) the crossdot product of tensors $\mathbf{A}$ and $\mathbf{B}$ is the vector

$\mathbf{A} \dot{\times} \mathbf{B} \equiv A_{i j} B_{m n}\left(\mathbf{e}_{i} \times \mathbf{e}_{m}\right)\left(\mathbf{e}_{j} \cdot \mathbf{e}_{n}\right)=A_{i j} B_{m j} \mathbf{e}_{i} \times \mathbf{e}_{j}$.
In Cartesian coordinates,

$$
\nabla \tilde{\mathbf{u}} \dot{\times} \nabla \mathbf{u}=\nabla \tilde{u}_{i} \times \nabla u_{i}=J_{i}\{\tilde{\mathbf{u}}, \mathbf{u}\} \mathbf{e}_{i} .
$$

This term involves the cross products of the gradients of the components along equal directions. Clearly, $\boldsymbol{A} \dot{\times} \boldsymbol{B}$ $=-\boldsymbol{B} \dot{\times} \boldsymbol{A}$, so that $\boldsymbol{A} \dot{\times} \boldsymbol{A}=\mathbf{0}$. Thus, when $\tilde{\mathbf{u}}=\mathbf{u}$ we have $\nabla \tilde{\mathbf{u}} \dot{\times} \nabla \mathbf{u}=\mathbf{0}, \tilde{\boldsymbol{\omega}}=\boldsymbol{\omega}=\boldsymbol{\nabla} \times \mathbf{u}, \tilde{\boldsymbol{\omega}}+\boldsymbol{\omega}^{p}$ is the absolute vorticity, and (3) reduces to the usual vorticity equation.

\section{2) RAte of CHANGE OF THE COFACTOR TENSOR $\overline{\nabla \tilde{\mathbf{u}}}$}

The cofactor tensor $\overline{\boldsymbol{A}}$ is defined so that $\overline{\boldsymbol{A}} \cdot \boldsymbol{A}^{\mathrm{T}}=$ $\boldsymbol{A}^{\mathrm{T}} \cdot \overline{\boldsymbol{A}}=\mathbf{I} \operatorname{det}(\boldsymbol{A})$, where $\mathbf{I}$ is the identity tensor $\mathbf{I}=\mathbf{i i}+$ jj $+\mathbf{k k}$. Coordinate expressions for the cofactor of the gradient of a vector are given in appendix $\mathrm{B}$. The rate of change of the cofactor of $\boldsymbol{\nabla} \tilde{\mathbf{u}}$ is obtained from (1),

$$
\begin{aligned}
\frac{d \overline{\nabla \tilde{\mathbf{u}}}}{d t}= & -\overline{\nabla \tilde{\mathbf{u}}} \boldsymbol{\nabla} \cdot \mathbf{u}+\nabla \mathbf{u}^{\mathrm{T}} \cdot \overline{\nabla \tilde{\mathbf{u}}}-\nabla \tilde{\mathbf{u}} \dot{\times} \nabla \mathbf{u} \omega^{p}+\tilde{\boldsymbol{\Gamma}} \cdot \nabla \mathbf{u} \\
& +\nabla \boldsymbol{\varphi} \stackrel{\times}{\times} \tilde{\mathbf{u}} .
\end{aligned}
$$

The proof of this expression, and of other tensor identities involving cofactors, is postponed to the next section. The first and second terms in the rhs are the divergence and tilting cofactor terms similar to those in the vorticity equation (3). The third and fourth terms include the dependence with the planetary vorticity $\boldsymbol{\omega}^{p}$.

The third term, $-\nabla \tilde{\mathbf{u}} \dot{\times} \nabla \mathbf{u} \boldsymbol{\omega}^{p}$, is identical to the tensor product of the third term in the vorticity Eq. (3) with $\boldsymbol{\omega}^{p}$, but with the opposite sign. In the fourth term, we have introduced $\tilde{\boldsymbol{\Gamma}}$, the skew-symmetric tensor associated to the vector $\nabla \tilde{\gamma}$, where $\tilde{\gamma} \equiv \tilde{\mathbf{u}} \cdot \boldsymbol{\omega}^{p}$ :

$$
\tilde{\boldsymbol{\Gamma}}=\mathbf{I} \times \nabla\left(\tilde{\mathbf{u}} \cdot \boldsymbol{\omega}^{p}\right)=\mathbf{I} \times \nabla \tilde{\gamma} .
$$

Thus $\tilde{\boldsymbol{\Gamma}} \cdot \boldsymbol{a}=\nabla \tilde{\gamma} \times \boldsymbol{a}$, which implies that the fourth term in (6) may be written also as

$$
\tilde{\boldsymbol{\Gamma}} \cdot \nabla \mathbf{u}=\nabla \tilde{\gamma} \times \nabla \mathbf{u}=\nabla\left(\tilde{\mathbf{u}} \cdot \boldsymbol{\omega}^{p}\right) \times \nabla \mathbf{u} .
$$

The last term in (6) expresses the dependence with the forcing $\boldsymbol{\varphi}$, and in Cartesian coordinates (appendix C) is

$$
\boldsymbol{\nabla} \boldsymbol{\varphi} \times \nabla \tilde{\mathbf{u}}=\nabla \varphi_{i} \times \nabla \tilde{u}_{j} \mathbf{e}_{i} \times \mathbf{e}_{j}=\mathbf{e}_{i} \times \mathbf{e}_{j} \boldsymbol{\varphi}_{, i} \times \tilde{\mathbf{u}}_{, j},
$$

where the comma-subindex notation indicates the partial derivative $\left(\chi_{, 1}, \chi_{, 2}, \chi_{, 3}\right)=\left(\chi_{, x}, \chi_{, y}, \chi_{, z}\right)=(\partial \chi / \partial x$, $\partial \chi / \partial y, \partial \chi / \partial z)$. This term involves the cross products of the gradients of the components of $\boldsymbol{\varphi}$ and $\tilde{\mathbf{u}}$ along different directions.

Using the identity

$$
(\mathbf{A} \times \mathbf{a}) \stackrel{\times}{\times} \mathbf{B}=(\mathbf{B} \cdot \mathbf{a}) \times \mathbf{A}+\mathbf{A} \dot{\times} \mathbf{B a},
$$

for any vector a, the terms involving $\boldsymbol{\omega}^{p}$ in (6) may be combined into

$$
\left(\nabla \mathbf{u} \times \boldsymbol{\omega}^{p}\right) \underset{\times}{\times} \nabla \tilde{\mathbf{u}}=\left(\nabla \tilde{\mathbf{u}} \cdot \boldsymbol{\omega}^{p}\right) \times \nabla \mathbf{u}-\nabla \tilde{\mathbf{u}} \dot{\times} \nabla \mathbf{u} \boldsymbol{\omega}^{p} .
$$


Therefore, (6) may be also expressed as

$$
\frac{d \overline{\nabla \tilde{\mathbf{u}}}}{d t}=-\overline{\nabla \tilde{\mathbf{u}}} \boldsymbol{\nabla} \cdot \mathbf{u}+\nabla \mathbf{u}^{\mathrm{T}} \cdot \overline{\nabla \tilde{\mathbf{u}}}+\nabla\left(\mathbf{u} \times \boldsymbol{\omega}^{p}+\varphi\right) \stackrel{\times}{\times} \boldsymbol{\nabla} \tilde{\mathbf{u}} .
$$

\section{3) Rate of Change of THE VORTICITY- VELOCITY GRADIENT COFACTOR TENSOR $\tilde{\Xi}$}

The vorticity-velocity gradient cofactor tensor is defined as

$$
\tilde{\Xi} \equiv \bar{\nabla} \tilde{\mathbf{u}}+\left(\tilde{\boldsymbol{\omega}}+\boldsymbol{\omega}^{p}\right) \boldsymbol{\omega}^{p} .
$$

Taking the tensor product of (3) with $\boldsymbol{\omega}^{p}$ and then adding it to (6), the term $\nabla \tilde{\mathbf{u}} \dot{\times} \nabla \mathbf{u} \boldsymbol{\omega}^{p}$ in both equations cancel. This results in the equation for the rate of change of $\tilde{\Xi}$,

$$
\frac{d \tilde{\Xi}}{d t}=-\tilde{\Xi} \boldsymbol{\nabla} \cdot \mathbf{u}+\nabla \mathbf{u}^{\mathrm{T}} \cdot \tilde{\Xi}+\boldsymbol{F},
$$

where the forcing tensor

$$
\boldsymbol{F} \equiv \tilde{\boldsymbol{\Gamma}} \cdot \nabla \mathbf{u}+\nabla \boldsymbol{\varphi} \times \underset{\times}{\times} \tilde{\mathbf{u}}+\nabla \times \varphi \boldsymbol{\omega}^{p} .
$$

4) RATE OF CHANGE OF THE SPECIFIC POTENTIAL $\tilde{\Xi}: \tilde{\Upsilon}$

Let the specific potential $\tilde{\Xi}$ (i.e., the specific potential vorticity-velocity gradient cofactor) be defined as the vector

$$
\tilde{\Upsilon} \equiv \alpha \nabla \theta \cdot \tilde{\Xi},
$$

where $\alpha \equiv \rho^{-1}$ is the specific volume so that $\tilde{\Upsilon}$ is an specific quantity (per unit of mass). The potential $\tilde{\Xi}$ density, that is, $\tilde{\Xi}$ per unit of volume, may be defined as $\hat{\tilde{r}} \equiv \nabla \theta \cdot \tilde{\Xi}$. The name potential is justified since $\tilde{r}$ can be defined in a more general form as a tensor if three, instead of only one, material invariants $\mathbf{X}=(X$, $Y, Z)$ are assumed to exist. In this case we may use $\mathbf{X}$ as the material coordinates so that the tensor $J \boldsymbol{\nabla} \mathbf{X} \cdot \tilde{\Xi}$, with $J^{-1}=\operatorname{det}(\boldsymbol{\nabla X})$ satisfying $\dot{J}=\boldsymbol{J} \cdot \mathbf{u}$, is a Piola transformation (see, e.g., Šilhavý 1997, p. 62). The tensor $J \boldsymbol{\nabla} \mathbf{X}_{\tilde{\tilde{E}}} \tilde{\Xi}$, if materially conserved, represents the value of $\tilde{\Xi}$ per unit of material volume, in the reference (e.g., initial) configuration. This three-dimensional generalization is identical to the case of PV (Viúdez 2001) and is not repeated here.

Using the identity for the rate of change of $\nabla \theta$,

$$
\frac{d \nabla \theta}{d t}=\nabla \dot{\theta}-\nabla \theta \cdot \nabla \mathbf{u}^{\mathrm{T}},
$$

and the mass conservation (2) in terms of $\alpha$,

$$
\dot{\alpha}=\alpha \boldsymbol{\nabla} \cdot \mathbf{u},
$$

the rate of change of $\Upsilon$ is

$$
\frac{d \tilde{\Upsilon}}{d t}=\alpha \nabla \theta \cdot \boldsymbol{F}+\alpha \nabla \dot{\theta} \cdot \tilde{\Xi} .
$$

Except for the use of the conservation of mass, Eq. (19) is a general mathematical identity since the vectors $\tilde{\mathbf{u}}$ and $\tilde{\varphi}$ and the scalar $\theta$ have not been defined. It is therefore independent of the SG assumptions, that is, independent of the definition of $\tilde{\mathbf{u}}$ as the geostrophic velocity and independent of the hydrostatic and $f$-plane approximations. An analogous result can be obtained if instead of the cofactor $\overline{\nabla \mathbf{u}}$ the transpose cofactor (or adjoint) $\overline{\nabla \mathbf{u}}^{\mathrm{T}}$ is used (appendix D).

Because of the identity $\boldsymbol{\nabla} \cdot \overline{\nabla \boldsymbol{a}}=\mathbf{0}$ for any vector $\mathbf{a}$, the potential $\tilde{\Xi}$ density may be also expressed in divergence form as

$$
\hat{\tilde{\Upsilon}} \equiv \nabla \cdot(\theta \tilde{\Xi}) .
$$

This implies that the rate of change of $\tilde{\Upsilon}$ (19) may be also expressed in different but equivalent forms using the divergence of tensors. This property is also common to the rate of change of PV [Viúdez 1999, his Eq. (23)].

In the next section we adopt the SG assumptions and show that these assumptions imply the conservation of the vertical component of $\tilde{\Upsilon}$ in the SG flow.

c. The rate of change of the vertical component of the geostrophic specific potential vorticityvelocity gradient cofactor tensor in the f-plane $S G$ theory

The $f$-plane hydrostatic SG theory assumes that

$$
\begin{aligned}
\boldsymbol{\varphi} & =-\alpha_{0} \nabla p-\alpha_{0} \rho g \mathbf{k}, \\
\boldsymbol{\omega}^{p} & =\zeta^{p} \mathbf{k}=f_{0} \mathbf{k}, \\
\tilde{\mathbf{u}}_{h} & =\mathbf{u}_{h}^{g}=\alpha_{0} f_{0} \mathbf{k} \times \nabla_{h} p, \\
\mathbf{k} \cdot \tilde{\mathbf{u}} & =\tilde{w}=w^{g}=0, \\
\dot{\theta} & =0 .
\end{aligned}
$$

Equation (21) introduces the pressure gradient term, where $\alpha_{0} \equiv \rho_{0}^{-1}$ is a constant specific volume, and $g \mathbf{k}$ is the acceleration due to gravity. In the $f$-plane geometry in Cartesian coordinates we adopt relation (22) where $f_{0}$ is the constant Coriolis parameter, and the northward planetary vorticity $\eta^{p}$ is ignored. Equations (23) and (24) are the definitions of the horizontal and vertical components of the geostrophic velocity, respectively. The vertical component of the dynamical equation (1) is therefore the hydrostatic relation. The symbols for density $\rho$ and constant specific volume $\alpha_{0}$ are used instead of potential temperature to make these results valid also in oceanic applications. Finally, (25) assumes that $\theta$ (the specific entropy, the potential temperature, or the density depending on the atmospheric or oceanic application) is materially conserved. 
The conditions (21)-(25) above imply that

$$
\begin{aligned}
\overline{\nabla \tilde{\mathbf{u}}} \cdot \mathbf{k} & =\overline{\nabla \mathbf{u}^{g}} \cdot \mathbf{k}=\nabla u^{g} \times \nabla \boldsymbol{v}^{g}, \\
\tilde{\boldsymbol{\omega}} & =\boldsymbol{\omega}^{g}=\left(\omega^{g}, \eta^{g}, \zeta^{g}\right)=\left(-\boldsymbol{v}_{z}^{g}, u_{z}^{g}, \boldsymbol{v}_{x}^{g}-u_{y}^{g}\right), \\
\tilde{\boldsymbol{\Xi}} & =\boldsymbol{\Xi}^{g}=\left[\nabla u^{g} \times \nabla \boldsymbol{v}^{g}+f_{0}\left(\boldsymbol{\omega}^{g}+f_{0} \mathbf{k}\right)\right] \mathbf{k} \equiv \boldsymbol{\xi}^{g} \mathbf{k}, \\
\tilde{\Upsilon} & =\Upsilon^{g}=\alpha \nabla \theta \cdot \boldsymbol{\xi}^{g} \mathbf{k} .
\end{aligned}
$$

Equation (26) expresses the vertical component of the cofactor of the geostrophic velocity gradient in terms of the three-dimensional gradients of the horizontal geostrophic components (Viúdez and Dritschel 2004), which is equivalent (appendix B) to the original form (Hoskins 1975) in terms of Jacobians:

$$
J_{y z}\left\{u^{g}, v^{g}\right\} \mathbf{i}+J_{z x}\left\{u^{g}, v^{g}\right\} \mathbf{j}+J_{x y}\left\{u^{g}, v^{g}\right\} \mathbf{k} .
$$

Expression (27) is the geostrophic vorticity in Cartesian coordinates, and (28) states that, in the SG theory, the geostrophic vorticity-velocity gradient cofactor tensor has only one vertical vector component, denoted here as $\boldsymbol{\xi}^{g}$. Finally, (29) states that, in the SG theory, the geostrophic vector $\mathrm{r}^{g}$ has only one scalar component, $\alpha \xi^{g} \cdot \nabla \theta$.

The SG conditions (21)-(25) imply also that the $\boldsymbol{\nabla} \theta$ component of the forcing tensor $\mathbf{F}(15)$ is null since

$$
\begin{aligned}
\tilde{\gamma} & =\tilde{\mathbf{u}} \cdot \boldsymbol{\omega}^{p}=w^{g} f_{0}=0 \\
(\nabla \varphi \times \nabla \tilde{\mathbf{u}}) \cdot \mathbf{k} & =\nabla \varphi \times \nabla \tilde{\boldsymbol{v}}-\nabla \psi \times \nabla \tilde{u} \\
& =-\alpha_{0}\left(\nabla p_{x} \times \nabla \boldsymbol{v}^{g}-\nabla p_{y} \times \nabla u^{g}\right)=\mathbf{0} \\
\nabla \theta \cdot \nabla \times \boldsymbol{\varphi} & =\alpha_{0} g \nabla \theta \cdot(\mathbf{k} \times \nabla \rho)=0 .
\end{aligned}
$$

Equation (30) is a consequence of the $f$-plane approximation and the horizontal nature of the geostrophic velocity. Result (31) is due to the definition of geostrophic velocity as the rotation of the horizontal pressure gradient, and (32) is due to the solenoidal property of the pressure gradient term and to the fact that $\theta$ is equal or proportional to $\rho$ [or that $\rho=f(\theta)$ ]. Therefore the vertical component of the specific potential geostrophic vorticity-velocity gradient cofactor,

$$
\Upsilon^{g} \equiv \Upsilon^{g} \cdot \mathbf{k}=\alpha \nabla \theta \cdot \xi^{g},
$$

is conserved under the SG assumptions,

$$
\frac{d \Upsilon^{g}}{d t}=0
$$

which is the scalar conservation equation of the SG flow (the SG PV equation).

\section{Tensor derivations of (6) and (14): The use of geostrophic coordinates}

This section provides a direct-tensor derivation of (6) and an alternative proof of (14). First, we supply some mathematical identities related to the rate of change of the gradient cofactor tensor.

\section{a. Mathematical identities related to the rate of change of the gradient cofactor tensor}

For any vector $\hat{\mathbf{u}}$, and using property (C4), the relation between the rates of change of $\overline{\nabla \hat{\mathbf{u}}}$ and $\boldsymbol{\nabla} \hat{\mathbf{u}}$ follows,

$$
\frac{d}{d t} \overline{\nabla \hat{\mathbf{u}}}=\left(\frac{d}{d t} \nabla \hat{\mathbf{u}}\right) \times \underset{\times}{\times} \hat{\mathbf{u}} .
$$

The first term in the rhs above is decomposed using the identify that commutes operators $d / d t$ and $\nabla$,

$$
\frac{d}{d t} \nabla \hat{\mathbf{u}}=\nabla \dot{\hat{\mathbf{u}}}-\nabla \mathbf{u} \cdot \nabla \hat{\mathbf{u}} .
$$

The following decomposition for a second rank tensor $\mathbf{A}$,

$$
\mathbf{A} \stackrel{\times}{\times} \mathbf{I}=\operatorname{tr}(\mathbf{A}) \mathbf{I}-\mathbf{A}^{\mathrm{T}},
$$

and the identity (appendix C)

$$
(\mathbf{A} \cdot \mathbf{B}) \stackrel{\times}{\times} \mathbf{B}=(\mathbf{A} \times \stackrel{\times}{\times} \mathbf{I}) \cdot \overline{\mathbf{B}},
$$

imply that

$$
(\mathbf{A} \cdot \mathbf{B}) \underset{\times}{\times} \mathbf{B}=\operatorname{tr}(\mathbf{A}) \overline{\mathbf{B}}-\mathbf{A}^{\mathrm{T}} \cdot \overline{\mathbf{B}} .
$$

Therefore,

$$
(\nabla \mathbf{u} \cdot \nabla \hat{\mathbf{u}}) \underset{\times}{\times} \nabla \hat{\mathbf{u}}=\nabla \cdot \mathbf{u} \overline{\nabla \hat{\mathbf{u}}}-\nabla \mathbf{u}^{\mathrm{T}} \cdot \overline{\nabla \hat{\mathbf{u}}},
$$

and the identity relating the rate of change of the cofactor of $\boldsymbol{\nabla} \hat{\mathbf{u}}$ with the gradient of the rate of change of û follows from (35):

$$
\frac{d}{d t} \overline{\nabla \hat{\mathbf{u}}}=-\overline{\nabla \hat{\mathbf{u}}} \nabla \cdot \mathbf{u}+\nabla \mathbf{u}^{\mathrm{T}} \cdot \overline{\nabla \hat{\mathbf{u}}}+\nabla \dot{\hat{\mathbf{u}}} \stackrel{\times}{\times} \nabla \hat{\mathbf{u}} .
$$

This identity is used in the next two subsections for particular choices of $\hat{\mathbf{u}}$.

\section{b. Derivation of (6)}

The rate of change of $\bar{\nabla} \tilde{\mathbf{u}}$ may be first obtained setting

$$
\hat{\mathbf{u}} \equiv \tilde{\mathbf{u}},
$$

so that the equation of motion is

$$
\dot{\hat{\mathbf{u}}}=-\boldsymbol{\omega}^{p} \times \mathbf{u}+\boldsymbol{\varphi} .
$$

Using $-\boldsymbol{\omega}^{p} \times \mathbf{u}=\mathbf{u} \times \boldsymbol{\omega}^{p}$, Eq. (12), and hence (6), follows directly from (41). 


\section{c. An alternative derivation of (14)}

Here we set

$$
\hat{\mathbf{u}} \equiv \tilde{\mathbf{u}}+\boldsymbol{\omega}^{p} \times \mathbf{r}
$$

so that the equation of motion is

$$
\dot{\hat{\mathbf{u}}}=\varphi \text {. }
$$

Above, $\mathbf{r}$ is the position vector of the fluid particle in the SG flow: $\dot{\mathbf{r}}=\mathbf{u}$. The gradient of $\hat{\mathbf{u}}$ is

$$
\boldsymbol{\nabla} \hat{\mathbf{u}}=\nabla \tilde{\mathbf{u}}+\mathbf{W}^{p},
$$

where $\mathbf{W}^{p}$ is the skew-symmetric tensor associated with the planetary vorticity vector $\boldsymbol{\omega}^{p}$,

$$
\begin{aligned}
\mathbf{W}^{p} & =\boldsymbol{\nabla}\left(\boldsymbol{\omega}^{p} \times \mathbf{r}\right)=\mathbf{I} \times \boldsymbol{\omega}^{p} \\
& =\boldsymbol{\omega}^{p} \mathbf{k} \wedge \mathbf{j}+\eta^{p} \mathbf{i} \wedge \mathbf{k}+\zeta^{p} \mathbf{j} \wedge \mathbf{i},
\end{aligned}
$$

so that, for any vector $\boldsymbol{a}$,

$$
\mathbf{W}^{p} \cdot \boldsymbol{a}=\boldsymbol{\omega}^{p} \times \boldsymbol{a} .
$$

Above $\mathbf{e}_{i} \wedge \mathbf{e}_{j}=\mathbf{e}_{i} \mathbf{e}_{j}-\mathbf{e}_{j} \mathbf{e}_{i}$ is the outer (exterior) product of vectors $\mathbf{e}_{i}$ and $\mathbf{e}_{j}$. The relation between the cofactors of $\boldsymbol{\nabla} \hat{\mathbf{u}}$ and $\boldsymbol{\nabla} \tilde{\mathbf{u}}$ may be obtained from relation (C1) and $\overline{\mathbf{W}^{p}}=\boldsymbol{\omega}^{p} \boldsymbol{\omega}^{p}$, yielding

$$
\overline{\nabla \hat{\mathbf{u}}}=\overline{\nabla \tilde{\mathbf{u}}}+\boldsymbol{\omega}^{p} \boldsymbol{\omega}^{p}+\nabla \tilde{\mathbf{u}}_{\times}^{\times} \mathbf{W}^{p} .
$$

The term $\nabla \tilde{\mathbf{u}} \times \mathbf{W}^{p}$ above is decomposed using the identity

$$
\boldsymbol{\nabla} \tilde{\mathbf{u}} \times \mathbf{W}^{p}=(\tilde{\boldsymbol{\omega}} \mathbf{l}-\mathbf{I} \times \nabla \tilde{\mathbf{u}}) \cdot \boldsymbol{\omega}^{p}
$$

so that

$$
\overline{\nabla \hat{\mathbf{u}}}=\overline{\nabla \tilde{\mathbf{u}}}+\left(\tilde{\boldsymbol{\omega}}+\boldsymbol{\omega}^{p}\right) \boldsymbol{\omega}^{p}-(\mathbf{I} \times \nabla \tilde{\mathbf{u}}) \cdot \boldsymbol{\omega}^{p}=\tilde{\Xi}-\tilde{\boldsymbol{\Gamma}} .
$$

From the identity (41) it follows that

$$
\begin{aligned}
\dot{\tilde{\Xi}}-\dot{\tilde{\Gamma}}= & -(\tilde{\Xi}-\tilde{\boldsymbol{\Gamma}}) \boldsymbol{\nabla} \cdot \mathbf{u}+\nabla \mathbf{u}^{\mathrm{T}} \cdot(\tilde{\Xi}-\tilde{\boldsymbol{\Gamma}})+\nabla \boldsymbol{\varphi} \\
& \times\left(\nabla \tilde{\mathbf{u}}+\mathbf{W}^{p}\right) .
\end{aligned}
$$

The rate of change of $\tilde{\boldsymbol{\Gamma}}$ is obtained using

$$
\frac{d}{d t} \nabla \tilde{\mathbf{u}}=-\nabla \mathbf{u} \cdot \nabla \tilde{\mathbf{u}}-\nabla\left(\boldsymbol{\omega}^{p} \times \mathbf{u}\right)+\nabla \boldsymbol{\varphi},
$$

which yields

$$
\frac{d \tilde{\boldsymbol{\Gamma}}}{d t}=\mathbf{I} \times\left(\frac{d}{d t} \nabla \tilde{\gamma}\right)=-\mathbf{I} \times \nabla \mathbf{u} \cdot \nabla \tilde{\gamma}+\mathbf{I} \times \nabla \boldsymbol{\varphi} \cdot \boldsymbol{\omega}^{p},
$$

where the term $\boldsymbol{\nabla}\left(\boldsymbol{\omega}^{p} \times \mathbf{u}\right) \cdot \boldsymbol{\omega}^{p}=-\left(\nabla \mathbf{u} \times \boldsymbol{\omega}^{p}\right) \cdot \boldsymbol{\omega}^{p}=$ $\mathbf{0}$ has been canceled. The last term $\mathbf{I} \times \boldsymbol{\nabla}\left(\varphi \cdot \boldsymbol{\omega}^{p}\right)$ represents the skew-symmetric tensor associated to the vector $\boldsymbol{\nabla}\left(\varphi \cdot \boldsymbol{\omega}^{p}\right)$. Using the identity (85) (see appendix E) it follows that the first term on the rhs of (54)

$$
-\mathbf{I} \times \nabla \mathbf{u} \cdot \nabla \tilde{\gamma}=\left(-\nabla \cdot \mathbf{u}+\nabla \mathbf{u}^{\mathrm{T}}\right) \cdot \tilde{\boldsymbol{\Gamma}}+\tilde{\boldsymbol{\Gamma}} \cdot \nabla \mathbf{u},
$$

so that we may finally express

$\dot{\tilde{\Gamma}}=\left(-\nabla \cdot \mathbf{u}+\nabla \mathbf{u}^{\mathrm{T}}\right) \cdot \tilde{\boldsymbol{\Gamma}}+\tilde{\boldsymbol{\Gamma}} \cdot \boldsymbol{\nabla} \mathbf{u}+\mathbf{I} \times \boldsymbol{\nabla}\left(\boldsymbol{\varphi} \cdot \boldsymbol{\omega}^{p}\right)$.

Using the same identity applied in (50) it follows that

$$
\begin{aligned}
\nabla \boldsymbol{\varphi} \times \stackrel{\times}{\times}\left(\nabla \tilde{\mathbf{u}}+\mathbf{W}^{p}\right)= & \nabla \boldsymbol{\varphi} \stackrel{\times}{\times} \boldsymbol{\nabla} \tilde{\mathbf{u}}+\boldsymbol{\nabla} \times \boldsymbol{\varphi} \boldsymbol{\omega}^{p} \\
& -\mathbf{I} \times \nabla \boldsymbol{\varphi} \cdot \boldsymbol{\omega}^{p},
\end{aligned}
$$

so that the term $\mathbf{I} \times \boldsymbol{\nabla} \boldsymbol{\varphi} \cdot \boldsymbol{\omega}^{p}$ cancels when $d \tilde{\boldsymbol{\Gamma}} / d t$ in (52) is given by (54), and (14) is obtained.

\section{d. Relation to the geostrophic coordinates}

Relation (51) provides an alternative interpretation of the SG invariant. Since under the SG assumptions on the $f$ plane

$$
\bar{\gamma}=\gamma^{g}=f_{0} w^{g}=0, \quad \tilde{\boldsymbol{\Gamma}}=\boldsymbol{\Gamma}^{g}=\mathbf{I} \times \nabla \gamma^{g}=0,
$$

it follows that

$$
\Xi^{g}=\overline{\nabla \hat{\mathbf{u}}^{g}},
$$

where $\hat{\mathbf{u}}^{g}=\mathbf{u}_{h}^{g}+f_{0} \mathbf{k} \times \mathbf{r}$, defined by (44) for the geostrophic flow, is an horizontal vector. Therefore the SG invariant may be alternatively interpreted as the scalar product of $\alpha \boldsymbol{\nabla} \theta$ with the cofactor of the gradient of $\tilde{\mathbf{u}}+$ $\boldsymbol{\omega}^{p} \times \mathbf{r}$ under the SG assumptions. In this case $\tilde{\mathbf{u}}=\mathbf{u}^{g}$, $\boldsymbol{\varphi}_{h}=\boldsymbol{\omega}^{p} \times \mathbf{u}_{h}^{g}$ with $\boldsymbol{\omega}^{p}=f_{0} \mathbf{k}$, and the horizontal component of (45) becomes

$$
\frac{d}{d t}\left[f_{0}\left(\mathbf{r}_{h}^{g}-\mathbf{r}_{h}\right)+\mathbf{k} \times \mathbf{u}_{h}^{g}\right]=0,
$$

where $\mathbf{r}_{h}^{g}$ is defined so that

$$
\frac{d \mathbf{r}_{h}^{g}}{d t} \equiv \mathbf{u}_{h}^{g}
$$

Expression (60) may be integrated yielding

$$
f_{0} \mathbf{r}_{h}^{g}=f_{0} \mathbf{r}_{h}-\mathbf{k} \times \mathbf{u}_{h}^{g},
$$

so that in Cartesian coordinates $\mathbf{r}_{h}^{g}(\boldsymbol{x}, t)=\left(x^{g}(\boldsymbol{x}, t), y^{g}(\boldsymbol{x}\right.$, $t))=\left(x+v^{g}(\boldsymbol{x}, t) / f_{0}, y-u^{g}(\boldsymbol{x}, t) / f_{0}\right)$ are the geostrophic coordinates (Yudin 1955). The gradient of $f_{0} \mathbf{r}_{h}^{g}$ is

$$
f_{0} \nabla \mathbf{r}_{h}^{g}=f_{0} \mathbf{I}_{h}+\nabla \mathbf{u}_{h}^{g} \times \mathbf{k}
$$

Since

$$
\begin{aligned}
\overline{f_{0} \mathbf{I}_{h}} & =f_{0}^{2} \mathbf{k k}=\mathbf{f}_{0} \mathbf{f}_{0}, \\
\overline{\nabla \mathbf{u}_{h}^{g} \times \mathbf{k}} & =\overline{\nabla \mathbf{u}_{h}^{g}}=\nabla u^{g} \times \nabla v^{g} \mathbf{k}, \\
\mathbf{I}_{h} \times\left(\nabla \mathbf{u}_{h}^{g} \times \mathbf{k}\right) & =\boldsymbol{\omega}^{g} \mathbf{f}_{0},
\end{aligned}
$$


where the geostrophic vorticity $\boldsymbol{\omega}^{g}=\nabla \times \mathbf{u}_{h}^{g}$, the cofactor

$$
\overline{f_{0} \boldsymbol{\nabla} \mathbf{r}_{h}^{g}}=\left(\boldsymbol{f}_{0}+\boldsymbol{\omega}^{g}\right) \mathbf{f}_{0}+\overline{\nabla \mathbf{u}_{h}^{g}} .
$$

The nonzero terms of the above expression are contained in the vector

$f_{0}^{2} \overline{\nabla \mathbf{r}_{h}^{g}} \cdot \mathbf{k}=\left(\mathbf{f}_{0}+\boldsymbol{\omega}^{g}\right) f_{0}+\overline{\nabla \mathbf{u}_{h}^{g}} \cdot \mathbf{k}=\Xi^{g} \cdot \mathbf{k} \equiv \boldsymbol{\xi}^{g}$.

The vertical component of the equation above is usually written in Cartesian components as

$$
J_{x y}\left\{x^{g}, y^{g}\right\}=f_{0}^{-1}\left(f_{0}+\omega^{g}\right)+f_{0}^{-2} J_{x y}\left\{u^{g}, v^{g}\right\},
$$

that is, in terms of the Jacobian $J_{x y}\left\{x^{g}, y^{g}\right\}$ of the geostrophic coordinates $\left(x^{g}, y^{g}\right)$.

\section{Concluding remarks}

This paper has introduced a new derivation and interpretation of the generalized SG material invariant in the theory of geophysical flows. The SG invariant is interpreted as the scalar vertical component of the specific potential vorticity-velocity gradient cofactor tensor. Under the SG assumptions (advection of the geostrophic velocity, hydrostatic, and $f$-plane approximations) this vertical component is materially conserved in the SG flow. The generalized SG invariant differs conceptually from the Beltrami-Rossby-Ertel specific potential vorticity.

The results obtained here address some of the questions recently collected by White (2002, section 9.4) relative to the SG theory, namely, "Can a version of SG having a more satisfactory definition of $\xi_{\mathrm{SG}}$ [where $\xi_{\mathrm{SG}}$ $=f_{0}+\zeta^{g}+f_{0}^{-1} J_{x y}\left\{u^{g}, v^{g}\right\}=f_{0}^{-1} \boldsymbol{\xi}^{g} \cdot \mathbf{k}$ in the notation used here] be derived?" (McIntyre and Roulstone 2002), and "Can SG be extended to the case of variable Coriolis parameter?" (Shutts 1980; Magnusdottir and Schubert 1991). In particular, the cancellation of the term $\boldsymbol{\nabla} \tilde{\mathbf{u}} \dot{\times} \nabla \mathbf{u} \boldsymbol{\omega}^{p}$ in the derivation of the rate of change of the specific potential $\tilde{\Xi}$, involves a whole tensor quantity and is therefore independent of the coordinate system. Thus, this cancellation takes place also in spherical coordinates and includes the beta term, or variable Coriolis parameter, since the beta term in the vorticity equation is the term involving the northward component of the planetary vorticity $\boldsymbol{\omega}^{p}$ (Viúdez 2003).

However, this is not the case of the source term involving the planetary vorticity in the equation for the rate of change of $\tilde{\Upsilon}$, that is, $\alpha \nabla \theta \cdot \nabla\left(\tilde{\mathbf{u}} \cdot \boldsymbol{\omega}^{p}\right) \times \nabla \mathbf{u}$. This term becomes zero because the northward planetary vorticity is neglected in the $f$-plane approximation, and the SG dynamics advects the horizontal geostrophic velocity, so that $\mathbf{u}^{g} \cdot \boldsymbol{\omega}^{p}=0$. This is not, in general, the case in spherical geometry or for three-dimensional flows $\tilde{\mathbf{u}}$. Thus, the material conservation of the SG invariant seems to be highly dependent on the SG assumptions, especially on the $f$-plane approximation and on the horizontal nature of the geostrophic velocity.
Acknowledgments. Support for this research has come from the Spanish Ministerio de Ciencia y Tecnología (Grant REN2002-01343). I thank two anonymous reviewers for their comments.

\section{APPENDIX A}

\section{Derivation of (3)}

Equation (3) may be derived in Cartesian components. The $\mathbf{e}_{i}$ component of the curl of the identity $d \tilde{\mathbf{u}} / d t$ $=\dot{\tilde{\mathbf{u}}}$ may be expressed as

$$
\begin{aligned}
\left(\frac{d \tilde{\omega}_{i}}{d t}\right) & =-\tilde{\omega}_{i} \boldsymbol{\nabla} \cdot \mathbf{u}+\tilde{\boldsymbol{\omega}} \cdot \nabla u_{i}+J_{i}\{\tilde{\mathbf{u}}, \mathbf{u}\}+\mathbf{e}_{i} \cdot(\boldsymbol{\nabla} \times \dot{\tilde{\mathbf{u}}}), \\
i & =1, \cdots, 3
\end{aligned}
$$

where the Jacobians $\left(J_{1}, J_{2}, J_{3}\right) \equiv\left(J_{y z}, J_{z x}, J_{x y}\right)$, and $J_{x y}\{\tilde{\mathbf{u}}, \mathbf{u}\} \equiv \tilde{\mathbf{u}}_{, x} \cdot \mathbf{u}_{, y}-\tilde{\mathbf{u}}_{, y} \cdot \tilde{\mathbf{u}}_{, x}$, etc. Since

$$
\nabla \dot{\tilde{\mathbf{u}}} \dot{\times} \nabla \mathbf{u}=J_{i}\{\tilde{\mathbf{u}}, \mathbf{u}\} \mathbf{e}_{i},
$$

and using the identity

$$
\boldsymbol{\nabla} \times\left(\boldsymbol{\omega}^{p} \times \mathbf{u}\right)=-\boldsymbol{\omega}^{p} \cdot \nabla \mathbf{u}+\boldsymbol{\omega}^{p} \boldsymbol{\nabla} \cdot \mathbf{u},
$$

Equation (3) is derived.

\section{APPENDIX B}

\section{The Vector Gradient Cofactor Tensor}

A vector $\mathbf{u}=(u, v, w)$ satisfies the identities

$$
\begin{aligned}
\overline{\nabla u} & =\nabla v \times \nabla w \mathbf{i}+\nabla w \times \nabla u \mathbf{j}+\nabla u \times \nabla v \mathbf{k} \\
& =\mathbf{i u}_{, y} \times \mathbf{u}_{, z}+\mathbf{j} \mathbf{u}_{, z} \times \mathbf{u}_{, x}+\mathbf{k} \mathbf{u}_{, x} \times \mathbf{u}_{, y} \\
& =\left\{\begin{array}{r}
J_{y z}\{v, w\} \mathbf{i i}+J_{y z}\{w, u\} \mathbf{i j}+J_{y z}\{u, v\} \mathbf{i k} \\
+J_{z x}\{v, w\} \mathbf{j i}+J_{z x}\{w, u\} \mathbf{j j}+J_{z x}\{u, v\} \mathbf{j k} \\
+J_{x y}\{v, w\} \mathbf{k i}+J_{x y}\{w, u\} \mathbf{k j}+J_{x y}\{u, v\} \mathbf{k k}
\end{array}\right\},
\end{aligned}
$$

where the Jacobians $J_{y z}\{a, b\} \equiv a_{, y} b_{, z}-a_{, z} b_{, y}$, etc. The determinant of the gradient tensor

$$
\operatorname{det}(\nabla \mathbf{u})=\boldsymbol{\nabla} u \cdot(\nabla v \times \nabla w)=\mathbf{u}_{, x} \cdot\left(\mathbf{u}_{, y} \times \mathbf{u}_{, z}\right) .
$$

\section{APPENDIX C}

\section{Tensor $A \times B$}

Second rank tensors $\mathbf{A}$ and $\mathbf{B}$ satisfy the identity involving cofactors

$$
\overline{\mathbf{A}+\mathbf{B}}=\overline{\mathbf{A}}+\overline{\mathbf{B}}+\mathbf{A} \times \underset{\times}{\times} .
$$

In Cartesian coordinates

$$
\begin{aligned}
\mathbf{A}_{\times}^{\times} \mathbf{B} & =A_{i j} B_{m n}\left(\mathbf{e}_{i} \mathbf{e}_{j}\right) \stackrel{\times}{\times}\left(\mathbf{e}_{m} \mathbf{e}_{n}\right) \\
& =A_{i j} B_{m n} \mathbf{e}_{i} \times \mathbf{e}_{m} \mathbf{e}_{j} \times \mathbf{e}_{n} .
\end{aligned}
$$


The double cross vector product symbol $\underset{x}{\times}$ and the cross-dot product symbol $(\dot{X})$ are here chosen in analogy to the double dot scalar product symbol (:) defined as

$$
\begin{aligned}
\mathbf{A}: \mathbf{B} & =A_{i j} B_{m n}\left(\mathbf{e}_{i} \mathbf{e}_{j}\right):\left(\mathbf{e}_{m} \mathbf{e}_{n}\right)=A_{i j} B_{m n} \mathbf{e}_{i} \cdot \mathbf{e}_{m} \mathbf{e}_{j} \cdot \mathbf{e}_{n} \\
& =A_{i j} B_{i j} .
\end{aligned}
$$

Since $\overline{c \mathbf{A}}=c^{2} \overline{\mathbf{A}}$ for any constant $c$ it follows from (C1) that

$$
\overline{\mathbf{A}}=\frac{1}{2} \mathbf{A} \times \mathbf{A} .
$$

For any vectors $\mathbf{a}=a_{i} \mathbf{e}_{i}$ and $\mathbf{b}=b_{i} \mathbf{e}_{i}$ it follows that

$$
\boldsymbol{\nabla} \mathbf{a} \times \nabla \mathbf{b}=\nabla a_{i} \times \nabla b_{j} \mathbf{e}_{i} \times \mathbf{e}_{j}=\mathbf{e}_{i} \times \mathbf{e}_{j} \mathbf{a}_{, i} \times \mathbf{b}_{, j} .
$$

Relation (38) can be proved using (C1) and the property $\overline{\mathbf{A} \cdot \mathbf{B}}=\overline{\mathbf{A}} \cdot \overline{\mathbf{B}}$,

$$
\begin{aligned}
(\mathbf{A} \cdot \mathbf{B}) \stackrel{\times}{\times} \mathbf{B} & =\overline{\mathbf{A} \cdot \mathbf{B}+\mathbf{B}}-\overline{\mathbf{A} \cdot \mathbf{B}}-\overline{\mathbf{B}} \\
& =\overline{(\mathbf{A}+\mathbf{I}) \cdot \mathbf{B}}-\overline{\mathbf{A}} \cdot \overline{\mathbf{B}}-\overline{\mathbf{B}} \\
& =(\overline{\mathbf{A}}+\mathbf{I}+\mathbf{A} \times \mathbf{I}) \cdot \overline{\mathbf{B}}-(\overline{\mathbf{A}}+\mathbf{I}) \cdot \overline{\mathbf{B}} \\
& =(\mathbf{A} \times \mathbf{I}) \cdot \overline{\mathbf{B}} .
\end{aligned}
$$

\section{APPENDIX D}

\section{The Transpose Cofactor Tensor $\overline{\nabla \tilde{u}}^{T}$}

The rate of change of the transpose cofactor (or adjoint) of $\boldsymbol{\nabla} \tilde{\mathbf{u}}$ is obtained from the transpose of Eq. (6), resulting in

$$
\begin{aligned}
\frac{d \bar{\nabla}^{\mathrm{T}}}{d t}= & -\bar{\nabla}^{\mathrm{T}} \boldsymbol{\nabla} \cdot \mathbf{u}+\bar{\nabla}^{\mathrm{T}} \cdot \nabla \mathbf{u}-\boldsymbol{\omega}^{p} \nabla \tilde{\mathbf{u}} \dot{\times} \nabla \mathbf{u} \\
& -\nabla \mathbf{u}^{\mathrm{T}} \dot{\tilde{\boldsymbol{\Gamma}}}+\nabla \varphi^{\mathrm{T}} \underset{\times}{\times} \nabla \tilde{\mathbf{u}}^{\mathrm{T}} .
\end{aligned}
$$

The rate of change of $\tilde{\Xi}^{\mathrm{T}} \equiv \bar{\nabla} \tilde{\mathbf{u}}^{\mathrm{T}}+\boldsymbol{\omega}^{p}\left(\tilde{\boldsymbol{\omega}}+\boldsymbol{\omega}^{p}\right)$ is obtained adding to (D1) the vorticity Eq. (3) (tensor product with $\boldsymbol{\omega}^{p}$ to cancel the term $\left.\boldsymbol{\omega}^{p} \nabla \tilde{\mathbf{u}} \dot{\times} \nabla \mathbf{u}\right)$, resulting in

$$
\frac{d \tilde{\Xi}^{\mathrm{T}}}{d t}=-\tilde{\Xi}^{\mathrm{T}} \boldsymbol{\nabla} \cdot \mathbf{u}+\tilde{\tilde{\Xi}}^{\mathrm{T}} \cdot \nabla \mathbf{u}+\mathbf{F}^{\mathrm{T}},
$$

where the transpose forcing tensor is

$$
\mathbf{F}^{\mathrm{T}}=-\nabla \mathbf{u}^{\mathrm{T}} \cdot \tilde{\boldsymbol{\Gamma}}+\nabla \boldsymbol{\varphi}^{\mathrm{T}} \times \underset{\times}{\times} \tilde{\mathbf{u}}^{\mathrm{T}}+\boldsymbol{\omega}^{p} \boldsymbol{\nabla} \times \boldsymbol{\varphi} .
$$

Since $\tilde{\Upsilon}=\alpha \tilde{\Xi}^{\mathrm{T}} \cdot \nabla \theta$, we may express (19) in terms of $\bar{\nabla}^{\mathrm{T}}$ as

$$
\frac{d \tilde{\Upsilon}}{d t}=\alpha \mathbf{F}^{\mathrm{T}} \cdot \nabla \theta+\alpha \tilde{\Xi}^{\mathrm{T}} \cdot \nabla \dot{\theta} .
$$

The adjoint tensor $\overline{\mathbf{A}}^{\mathrm{T}}$ satisfies the relation $\overline{\mathbf{A}}^{\mathrm{T}} \cdot \mathbf{A}=$ $\mathbf{A} \cdot \overline{\mathbf{A}}^{\mathrm{T}}=\operatorname{det}(\mathbf{A}) \mathbf{I}$. If $\operatorname{det}(\mathbf{A}) \neq 0$ then the inverse tensor $\mathbf{A}^{-1}$ exists, $\mathbf{A}^{-1}=\overline{\mathbf{A}}^{\mathrm{T}} / \operatorname{det}(\mathbf{A})$. The existence of the in- verse tensor $(\nabla \tilde{\mathbf{u}})^{-1}$ is not however assumed in this work since in the case of the geostrophic velocity $\tilde{w}=$ $w^{g}=0$, which implies that $\operatorname{det}(\boldsymbol{\nabla} \tilde{\mathbf{u}})=0$.

\section{APPENDIX E}

\section{Proof of (55)}

Let $\mathbf{A}$ be second rank tensor, and $\mathbf{V}$ and $\mathbf{A}_{v}$ be the skew-symmetric tensors related to the vectors $\mathbf{v}$ and $\mathbf{A} \cdot \mathbf{v}$, that is, $\mathbf{V}=\mathbf{I} \times \mathbf{v}$ and $\mathbf{A}_{\boldsymbol{v}}=\mathbf{I} \times(\mathbf{A} \cdot \mathbf{v})$, respectively. It follows that

$$
\mathbf{A}_{v}=\operatorname{tr}(\mathbf{A}) \mathbf{V}+\left\{(\mathbf{V} \cdot \mathbf{A})^{\mathrm{T}}-(\mathbf{V} \cdot \mathbf{A})\right\}
$$

where the three terms above are clearly skew symmetric. Since $\mathbf{V}^{\mathrm{T}}=-\mathbf{V}$ and rearranging,

$$
\mathbf{V} \cdot \mathbf{A}=\left[\operatorname{tr}(\mathbf{A}) \mathbf{I}-\mathbf{A}^{\mathrm{T}}\right] \cdot \mathbf{V}-\mathbf{A}_{\boldsymbol{v}}
$$

Using (50) it follows that

$$
\mathbf{V} \cdot \mathbf{A}=(\mathbf{A} \times \stackrel{\times}{\times} \mathbf{I}) \cdot \mathbf{V}-\mathbf{A}_{\boldsymbol{v}} .
$$

\section{REFERENCES}

Beltrami, E., 1871: Sui principii fondamentali dell'idrodinamica razionali. Memorie della Academia delle Scienze dell'Instituto di Bologna, Serie 3, Vol. 1, 431-476.

Bridges, T. J., P. E. Hydon, and S. Reich, 2005: Vorticity and symplecticity in Lagrangian fluid dynamics. J. Phys. A.: Math. Gen., 38, 1403-1418.

Casey, J., and P. M. Naghdi, 1991: On the Lagrangian description of vorticity. Arch. Ration. Mech. Anal., 115, 1-14.

Ehrendorfer, M., 2004: A vector derivation of the semigeostrophic potential vorticity equation. J. Atmos. Sci., 61, 1461-1466.

Eliassen, A., 1949: The quasi-static equations of motion with pressure as independent variable. Geofys. Publ., 17, 1-44.

Ertel, H., 1942: Ein neuer hydrodynamischer Wirbelsatz. Meteor. Z., 59, 277-281.

Fjortoft, R., 1962: On the integration of a system of geostrophically balanced prognostic equations. Proc. Int. Symp. Numerical Weather Prediction, Tokyo, Japan, Meteorological Society of Japan, 153-159.

Hoskins, B. J., 1975: The geostrophic momentum approximation and the semi-geostrophic equations. J. Atmos. Sci., 32, 233242.

_ , and I. Draghici, 1977: The forcing of ageostrophic motion according to the semi-geostrophic equations and in an isentropic coordinate model. J. Atmos. Sci., 34, 1859-1867.

Magnusdottir, G., and W. Schubert, 1991: Semi-geostrophic theory on the hemisphere. J. Atmos. Sci., 48, 1449-1456.

McIntyre, M. E., and I. Roulstone, 2002: Are there higheraccuracy analogues to semigeostrophic theory? Geometric Methods and Models. Vol. 2, Large-Scale Atmosphere-Ocean Dynamics, J. Norbury and I. Roulstone, Eds., Cambridge University Press, 301-364.

Rossby, C.-G., 1940: Planetary flow patterns in the atmosphere. Quart. J. Roy. Meteor. Soc., 66 (Suppl.), 68-87.

Schröder, W., 1991: Geophysical hydrodynamics and Ertel's potential vorticity. Selected Papers of Hans Ertel, Interdivisional Commission on History of International Association for Geomagnetism and Aeronomy, 218 pp.

Shutts, G. J., 1980: Angular momentum coordinates and their use in zonal geostrophic motion in the hemisphere. J. Atmos. Sci., 37, 1126-1132.

Šilhavý, M., 1997: The Mechanics and Thermodynamics of Continuous Media. Springer, 504 pp. 
Viúdez, A., 1999: On Ertel's potential vorticity theorem. On the impermeability theorem for potential vorticity. J. Atmos. Sci., 56, 507-516.

2001: The relation between Beltrami's material vorticity and Rossby-Ertel's potential vorticity. J. Atmos. Sci., 58, 25092517.

2003: A new interpretation of the beta term in the vorticity equation. J. Atmos. Sci., 60, 1866-1870.

, and D. G. Dritschel, 2004: Potential vorticity and the quasi- geostrophic and semigeostrophic mesoscale vertical velocity. J. Phys. Oceanogr., 34, 865-887.

White, A. A., 2002: A view of the equations of meteorological dynamics and various approximations. Analytical Methods and Numerical Models. Vol. 1, Large-Scale AtmosphereOcean Dynamics, J. Norbury and I. Roulstone, Eds., Cambridge University Press, 1-100.

Yudin, M. I., 1955: Invariant quantities in large-scale atmospheric processes (in Russian). Glav. Geofiz. Observ., 55, 3-12. 